\title{
Desarrollo de la versión móvil para la red social FEIBook
}

\author{
Luis Abraham Zavaleta Ibarra, Gerardo Contreras Vega, Karen Cortés Verdín, Juan \\ Carlos Pérez Arriaga \\ Universidad Veracruzana, Facultad de Estadística e Informática, \\ Xalapa, Ver., México
}

luis.ibarra310@gmail.com,gcontreras@uv.mx,kcortes@uv.mx, juaperez@uv.mx

\begin{abstract}
Resumen. Las redes sociales han transformado la manera en como las personas se comunican y se relacionan en la actualidad. Su impacto ha sido tan grande que cada vez es más común observar a las personas haciendo uso de las redes sociales. Actualmente se tiene claro que las redes sociales han llegado para quedarse y que su presencia es cada vez más habitual, pero el acceso a las redes sociales a través de los dispositivos móviles dentro del ámbito educativo es un tema que muy pocos han abordado en la actualidad. Por otra parte, hoy en día los dispositivos móviles ofrecen a los usuarios muchas funcionalidades y características que hace pocos años solo era posible encontrar en equipos más complejos y costoso, como las computadoras de escritorio o laptops. Haciendo uso de las redes sociales en combinación con tecnología móvil es posible crear aplicaciones para su uso en el ámbito universitario. La Facultad de Estadística e Informática de la Universidad Veracruzana desarrolló recientemente una red social para el seguimiento de egresados denominada FEIBook. Con esta red se busca mantener contacto con los egresados y que éstos proporcionen información de su desempeño laboral para realizar los estudios que correspondan. Es así, que en búsqueda de obtener una mayor participación en la red social, se desarrolla la versión móvil de dicha red.
\end{abstract}

Palabras Clave: Redes sociales, Seguimiento de Egresados, Android, Servicios Web, Móviles.

\section{$1 \quad$ Introducción}

El estudio de seguimiento de egresados es una estrategia evaluativa que posibilita conocer la situación profesional o laboral de un egresado de una carrera profesional con la finalidad del mejoramiento institucional [1]. Los estudios de seguimiento de egresados son importantes ya que permiten conocer la situación profesional del egresado. Con esta información se determina la pertinencia del programa educativo, se actualizan los progra- 
mas de estudio y se evalúa la calidad de las estrategias educativas y del cuerpo docente de la institución.

Estudios realizados en el año 2012 por la Salzburg Academy of Global Media Literacy [12] revelan que México es el segundo país del mundo con más usuarios universitarios conectados en redes sociales, con un promedio de tres horas diarias. Se destaca que el $66 \%$ de los estudiantes reportan el uso de teléfonos celulares y teléfonos tipo Smartphone para conectarse a las redes sociales, bajando así el uso de computadoras de escritorio e incluso laptops. El artículo asegura que publicar estados y comentar actualizaciones y contenidos de amigos son las actividades más realizadas por los usuarios universitarios.

En la Facultad de Estadística e Informática (FEI) de la Universidad Veracruzana se ha pensado resolver la necesidad del seguimiento de egresados mediante el uso de redes sociales y de la tecnología móvil. Actualmente ya se tiene una red social, que lleva por nombre FEIBook [2][8][9]. Esta red social tiene como objetivo principal crear un vínculo con los egresados de la FEI para así crear un directorio actualizado de egresados. Algunas de las actividades que puede realizar actualmente un usuario de FEIBook son: compartir lo que están pensando, agregar fotografías, agregar o modificar su perfil de usuario, comentar una publicación, hacer amistad con usuarios de la red y enviar mensajes privados a sus amigos.

Uno de los problemas actuales de FEIBook es el desinterés de los egresados en actualizar sus datos y situación laboral. Es por esto que se decidió realizar una versión móvil de la actual red social y es así que se desarrolla una versión de FEIBook para dispositivos móviles que cuenten con Sistema Operativo Android [13].

Este documento presenta el desarrollo de FEIBook versión móvil y se encuentra organizado de la siguiente manera: el apartado dos describe las redes sociales móviles, tipos y su aplicación en diversos contextos. A continuación, se describe FEIBook. En el cuarto apartado se presenta el desarrollo de FEIBook móvil, incluyendo las pruebas realizadas. Por último, se presentan las conclusiones y se describen los trabajos futuros.

\section{Redes sociales móviles}

Las redes sociales móviles son un medio de comunicación que mezcla la tecnología móvil y la infraestructura de Internet. Las redes sociales móviles (Mobile Social Networks) o MSN hacen referencia a todos los elementos que permiten el desplazamiento, carga y consumo de los sitios de redes sociales alojados en la web a través de un dispositivo móvil. Las redes sociales móviles pueden ser divididas en cinco tipos diferentes[11]:

- Los Medios Sociales son redes sociales móviles no profesionales para subir fotos digitales, mensajes escritos y documentos (ej. Blogs), sonido y vídeo.

- Los Perfiles son páginas de contenido dinámico que pueden ser actualizados por el autor, mejorando así la interacción entre el usuario y la red social. 
- Los Portales de Comunicación son aquellos destinados a que un usuario se suscriba a una aplicación para obtener el contenido de un grupo o comunidad.

- Las Comunidades, dentro del contexto de las redes sociales móviles, se definen como comunidades de lazos interpersonales que promueven la sociabilidad, apoyo, información, sentido de pertenencia e identidad social.

- La Mensajería Social para redes sociales móviles se refiere a una herramienta libre que permite a las personas intercambiar mensajes con grupos (comunidades) o particulares, en ocasiones combinando mensajes de texto plano, pero casi siempre utilizando la plataforma web y el navegador.

Con el fin de tener servicios que cubran las necesidades de los usuarios de redes sociales móviles y evitar las interfaces de usuario excesivamente complejas o redundantes, los creadores de las comunidades móviles deben identificar las necesidades de una sociedad. Por lo regular una red social móvil atiende un conjunto específico de necesidades, aunque en ocasiones los servicios de una RSM logran satisfacer más necesidades de una comunidad, esto es debido a los servicios que implementan [11].

El entretenimiento y la curiosidad son necesidades a cubrir en las RSM, mismas que se complementan con la necesidad de hacer amigos y pertenecer a un grupo. Algunas redes sociales móviles buscan o promueven la descarga de contenido, la compra o venta de artículos, la competencia entre los usuarios y en ocasiones generar ingresos directos o indirectos para los usuarios. Las comunidades de entretenimiento casi siempre permiten a los usuarios compartir sus experiencias y recomendaciones mediante votos o comentarios.

Una RSM puede ayudar a sus miembros a desarrollarse y crecer profesionalmente. Existen comunidades móviles diseñadas para el intercambio y presentación de información referente a la formación profesional y laboral. De esta manera las comunidades móviles cubren las necesidades profesionales de la comunidad. Un ejemplo es la red social LinkedIn.

\section{FEIBook}

FEIbook es la red social de seguimiento de egresados de la Facultad de Estadística e Informática [2][13]. Elgg [4]es el CMS que soporta el desarrollo de FEIBook. Elgg fue creado por Ben Wedmuller y David Tosh. Elgg crece y mejora gracias al desarrollo comunitario de programadores en todo el mundo. En agosto de 2008 recibió el premio como la mejor plataforma social de trabajo [10]. Elgg sigue el patrón Modelo Vista Controlador (MVC) [3]. En este patrón, el modelo es un conjunto de clases que define la forma en la que funciona la plataforma y almacena en la base de datos. La vista maneja la interfaz del usuario. El controlador es el intermediario entre la vista y el modelo, recibe las órdenes del usuario a través de la vista, traduce dichas instrucciones al modelo y recibe la respuesta de éste para enviarla a la vista. 
Para el desarrollo de FEIBook, se adaptó Elgg conforme a los requisitos para la red social de la FEI: publicar fotos, publicar un cambio de estado, enviar mensajes privados, compartir archivos, chat entre usuarios de la red, crear y modificar perfil para cada usuario, crear grupos privados y compartir contenidos con otras redes sociales. La adaptación de Elgg se llevó a cabo mediante el desarrollo de plugins. Los plugins que son fáciles de incorporar gracias a la arquitectura tipo MVC de Elgg. Un plugin es una aplicación que se agrega o se elimina hasta obtener todas las funcionalidades deseadas de Elgg [6]. Todos los plugins que se anexan a Elgg también deben seguir el estilo MVC. A continuación, se explican cada uno de los elementos de Elgg.

\subsection{El modelo de Elgg}

El modelo de Elgg recibe el nombre de core Elgg o núcleo. Dicho core está diseñado en unidades atómicas llamadas unidades. Las clases más empeladas y las más importantes, son las siguientes [5]:

- ElggEntity: Representa cualquier elemento de la red social, se encarga de otorgar los permisos de acceso, almacena el usuario propietario de esa entidad, asigna el identificador principal.

- ElggObject: Esta entidad representa todos los objetos arbitrarios como: los archivos subidos, los favoritos de los usuarios, los mensajes publicados.

- ElggUser: Se encarga de los usuarios, sus datos y permisos.

- ElggSite: La entidad representa los datos de la red social como el nombre, la dirección Web, el dominio.

- ElggGroup: Controla los grupos de usuarios creados.

- Metadatos: Contiene información que describe un objeto por ejemplo las rutas de los archivos.

- Anotaciones: Contiene información general agregada por terceros, como lo son los comentarios de una publicación.

- ElggRelationship: Se encarga de unir las entidades de acuerdo a su interacción, por ejemplo la pertenencia de un usuario a un grupo.

\subsection{La vista de Elgg}

Las vistas de Elgg se encargan de mostrar desde el diseño de las páginas hasta los formularios en el navegador y también se pueden realizar vistas avanzadas. Algunos ejemplos de vistas avanzadas son [5]: HTML estándar, HTML optimizado para accesibilidad, widgets embebidos e Internet móvil. 


\subsection{El controlador de Elgg}

El controlador dentro de Elgg corresponde a Elgg Action. Una acción es el código que se ejecuta cuando un usuario realiza algo dentro de Elgg[7]. Se considera acción a cualquier actividad que puede realizar el usuario como iniciar sesión, crear y/o eliminar un comentario. La acción procesa el formulario o petición enviada por el usuario, realiza los cambios necesarios en la base de datos y envía una respuesta al usuario, proporcionando de esta manera la interactividad del usuario con el modelo. Todas las acciones se desarrollan en scripts PHP por separado y el nombre del archivo debe ser del mismo nombre que la acción que puede realizar. Todas las acciones son ejecutadas por un controlador central llamado action_handler.

\subsection{Elgg realización de plugins}

El core de Elgg proporciona las funcionalidades básicas para una red social, sin embargo, el administrador tiene la posibilidad de construir la red social en base a sus necesidades, lo cual se logra mediante el uso de plugins. Los plugins permiten agregar nuevas funcionalidades, por ejemplo agregar un chat, un blog e inclusive cambiar toda la vista de Elgg. Estos plugins son reconocidos por el core después de colocarlos dentro del directorio $/ m o d$, pero no es hasta que son configurados por el administrador que serán visibles en la red social. Los plugins son reconocidos por el core sólo si están desarrollados bajo las pautas designadas por Elgg [6] y si han sido creados siguiendo el patrón MVC. Las pautas para desarrollar un plugin para Elgg son las siguientes: 1) Conservar el esqueleto o estructura por defecto, el cual puede consultarse en la documentación de Elgg; 2) Conservar los nombres y 3) Seguir el MVC de Elgg.

\section{Desarrollo de FEIBook móvil}

La finalidad principal de hacer una versión móvil de la red social de la FEI es dar a los egresados la oportunidad de acceder desde cualquier dispositivo móvil con sistema operativo Android. Así, la FEI podrá contar con información necesaria para el seguimiento de egresados. El desarrollo de la versión móvil de FEIBook, consistió en la creación y uso del API FEIBook_WebServices para la comunicación entre el cliente y el servidor, así como en la creación de una aplicación móvil para los dispositivos con sistema operativo Android. A continuación, se explica con mayor detalle el desarrollo.

\subsection{Plugin FEIBook_WebServices}

Elgg permite exponer algunas funcionalidades para poder ser consumidas por aplicaciones de terceros. Por otra parte, dentro de la comunidad de Elgg encuentran algunos plugins de 
Servicios Web que logran exponer algunas funcionalidades. Sin embargo, después de analizar y probar estos, se llegó a la conclusión que ninguno cubría las necesidades de FEIBook móvil o estaban creados para otras versiones de Elgg y no eran compatibles con FEIBook. Es así como se decide crear un plugin propio que cubra las necesidades requeridas y que sea compatible con la versión de Elgg 1.7.17 la cual está siendo usada en FEIBook. Este plugin se denomina FEIBook_Webservices.

Los Servicios Web surgen por la necesidad de ofrecer aplicaciones distribuidas haciendo uso de software que pueda ser ejecutado en diferentes sistemas operativos y arquitecturas, utilizando diferentes lenguajes de programación y herramientas.

Actualmente existen varias técnicas de arquitectura software para la creación de servicios web: RPC (Remote Procedure Call), SOA (Service Oriented Architecture) y REST (REpresentational State Transfer). Se elige REST para la implementación del plugin ya que se pueden trabajar sistemas de hipermedia distribuidos como la Web y por su funcionamiento independiente que garantiza la independencia entre servidores y clientes. REST, se basa en estándares como HTTP, URI y XML, entre otros.

FEIBook_WebServices, es un plugin que únicamente usa el controlador de Elgg. Es así, que sirve como una interfaz entre el cliente móvil y el servidor. De esta manera, recibe las peticiones del cliente y responde estas peticiones con base en los criterios establecidos por el propio Elgg core.

Los pasos seguidos para la construcción del plugin son los siguientes:

1. Se crea un nuevo directorio dentro del directorio mod de Elgg. El directorio creado lleva por nombre FEIBook_WebServices y con este nombre será identificado y reconocido por el motor de Elgg.

2. Se crea el archivo manifest.xml dentro del directorio FEIBook_WebServices, este archivo contiene información del plugin como nombre del plugin, autor, versión, licencia, entre otros datos que pueden ser añadidos.

3. Dentro del directorio FEIBook_WebServices se crea un nuevo archivo que lleva por nombre start.php. Este archivo contiene las funciones que serán expuestas mediante la función expose_function(), y que permite a otras aplicaciones interactuar con la red social.

Posteriormente, se debe comprobar que el núcleo de Elgg reconoce plugin y que fue habilitado correctamente. Para esto, se ingresa a FEIBook con una cuenta de administrador $\mathrm{y}$, en la sección de herramientas de administración, se habilita el plugin FEIBook_WebServices que, por defecto, aparece deshabilitado. Entonces, Elgg notifica con un mensaje si el plugin fue reconocido y habilitado correctamente.

Al agregar el plugin FEIBook_WebServices se pone una capa al MVC de Elgg (FEIBook), modificando así su arquitectura. En la figura 1, en la siguiente página, se presenta la arquitectura modificada empleando un diagrama de paquetes de UML. Se puede observar como el plugin FEIBook_WebServices interactúa con el controlador, dando así acceso a la funcionalidad de FEIBook. 


\subsection{Aplicación FEIBook móvil}

La aplicación FEIBook móvil es la responsable de consumir las funcionalidades expuestas por el plugin FEIBook_WebServices. Por cuestiones de tiempo, se opta por desarrollar esta aplicación móvil en Android, ya que es un sistema operativo popular con un SDK fácil de implementar y de utilizar.

Android es un sistema operativo libre que no necesita ningún tipo de hardware especial para desarrollar aplicaciones. Actualmente Android tiene mayor popularidad y demanda en comparación con iOS y es más común observar a estudiantes y maestros de la FEI utilizar dispositivos con esta plataforma.

Para que el usuario pueda acceder a las opciones ofrecidas por la aplicación móvil de FEIBook, debe hacer uso de un menú de navegación llamado Navigation Drawer, ubicado en el lado izquierdo de la pantalla. El menú de navegación de FEIBook móvil cuenta con las siguientes opciones:

- El Muro.

- Mi Perfil.

- Amigos.

- Buscar personas.

- Mensajes.

- Ayuda.

- Cerrar sesión.

- Notificaciones.

El intercambio de información entre la aplicación FEIBook y el servidor se realiza mediante el protocolo HTTP. Además, FEIBook móvil hace uso de SSL para mantener la seguridad cuando un usuario accede a la red social. Para poder hacer uso del certificado dentro de la aplicación móvil de FEIBook y tener una conexión segura se prepara el certificado y se añade a la aplicación, para que esta pueda hacer uso de él al momento de realizar una conexión HTTPS. FEIBook móvil conecta con el servidor de FEIBook para poder realizar las tareas que el usuario solicita. Además, realiza peticiones de manera periódica para poder notificar al usuario de los cambios y noticias más recientes en la red social. Esta comunicación se realiza a través de Internet ya sea mediante una conexión Wi-Fi o por datos móviles. 
Luis Abraham Zavaleta Ibarra, Gerardo Contreras Vega, Karen Cortés Verdín, et al.

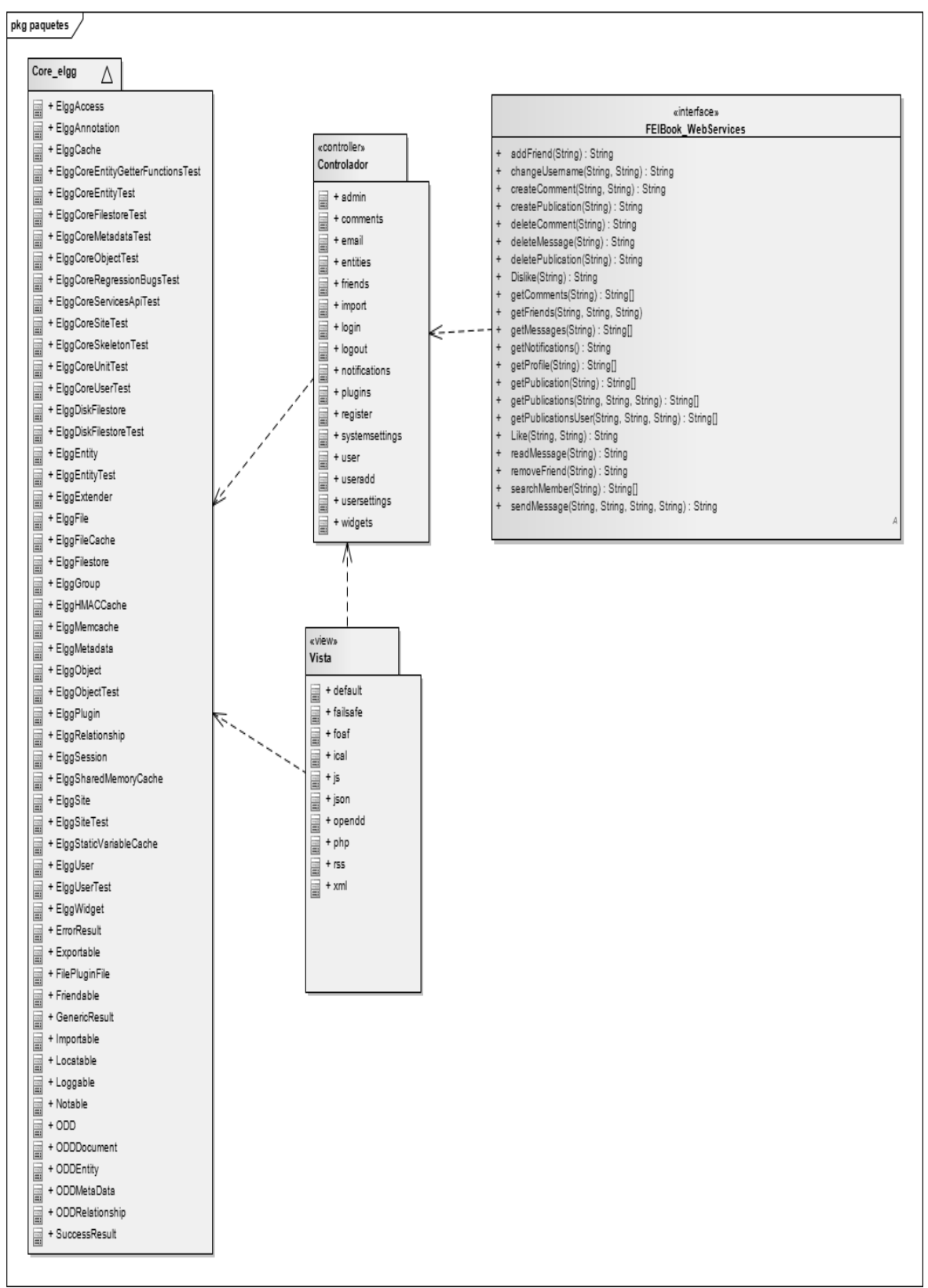

Fig. 1. Arquitectura de FEIBook modificada. 


\subsection{Pruebas}

Las pruebas realizadas a FEIBook móvil se basan en casos de uso [13]. El proceso de pruebas consideró los tres pasos fundamentales a realizar: entorno, desarrollo y ejecución. En la tabla 1, abajo, se presentan los 11 casos de prueba de la aplicación, para realizar las pruebas, se subió a un servidor remoto una copia de la red FEIBook con las mismas características que la versión en producción. Participaron 15 estudiantes de la FEI, realizándose las pruebas en dos días. Cada estudiante contó con dos horas para realizar los casos de pruebas planteados. Las versiones de los sistemas operativos Android variaba de la 4.0.4 a la 4.4.2. La versión más común, con 7 dispositivos, fue la 4.1.2.

Tabla 1. Casos de prueba

\begin{tabular}{|c|c|c|}
\hline ID & Nombre del caso de prueba & Propósito de la prueba \\
\hline P1 & Instalar la aplicación. & $\begin{array}{l}\text { La aplicación FEIBook móvil puede ser descargada } \\
\text { del servidor e instalada en el dispositivo móvil. }\end{array}$ \\
\hline $\mathrm{P} 2$ & $\begin{array}{l}\text { Ingresar a FEIBook móvil con } \\
\text { datos erróneos. }\end{array}$ & $\begin{array}{l}\text { Se impide el acceso a usuarios que proporcionan } \\
\text { datos incorrectos. }\end{array}$ \\
\hline P3 & Ingresar a FEIBook móvil. & $\begin{array}{l}\text { Los usuarios ingresan cuando proporcionan nombre } \\
\text { de usuario y contraseña correctos. }\end{array}$ \\
\hline P4 & Publicar en el muro. & $\begin{array}{l}\text { La aplicación permite a los usuarios realizar publi- } \\
\text { caciones en el muro de FEIBook. }\end{array}$ \\
\hline P5 & Comentar a una publicación. & Los usuarios comentan las publicaciones del muro. \\
\hline P6 & $\begin{array}{l}\text { Agregar a un usuario como } \\
\text { amigo. }\end{array}$ & $\begin{array}{l}\text { Buscar un miembro de la red social FEIBook y que } \\
\text { es posible agregarlo a su lista de amigos. }\end{array}$ \\
\hline P7 & Enviar mensaje a un usuario. & $\begin{array}{l}\text { Los usuarios pueden enviar mensajes privados a } \\
\text { otros usuarios. }\end{array}$ \\
\hline P8 & Revisar mensaje privado. & $\begin{array}{l}\text { El usuario puede revisar sus mensajes privados y } \\
\text { puede responder a un mensaje enviado por otro } \\
\text { usuario. }\end{array}$ \\
\hline P9 & $\begin{array}{l}\text { Eliminar comentario de una } \\
\text { publicación. }\end{array}$ & $\begin{array}{l}\text { Comprobar que el usuario puede eliminar los co- } \\
\text { mentarios hechos en una publicación }\end{array}$ \\
\hline P10 & $\begin{array}{l}\text { Eliminar usuario de la lista de } \\
\text { amigos. }\end{array}$ & $\begin{array}{l}\text { La aplicación FEIBook permite a los usuarios eli- } \\
\text { minar usuario de FEIBook de su lista de amistades. }\end{array}$ \\
\hline P11 & $\begin{array}{l}\text { Cerrar sesión en FEIBook mó- } \\
\text { vil }\end{array}$ & $\begin{array}{l}\text { Comprobar que el usuario puede cerrar la sesión } \\
\text { creada en la aplicación móvil de FEIBook. }\end{array}$ \\
\hline
\end{tabular}


En la tabla 2, abajo, se listan los casos de prueba con resultados fallidos.

Tabla 2. Casos de prueba con resultados fallidos.

\begin{tabular}{ccl}
\hline ID & $\begin{array}{c}\text { \# re- } \\
\text { sultados } \\
\text { fallidos }\end{array}$ & \\
\hline P4 & 1 & $\begin{array}{l}\text { Un usuario reportó: "La aplicación se detuvo y nunca cargó el muro. } \\
\text { Después de volver a abrir la aplicación cargó el muro y se pudo publi- } \\
\text { car". } \\
\text { Seis usuarios reportaron el error: "La aplicación FEIBook se detuvo". } \\
\text { Un usuario comento: "No aparece el cesto de basura" } \\
\text { Un usuario comentó: "Se borró el comentario pero no hubo ningún } \\
\text { diálogo". } \\
\text { Siete usuarios reportaron: "Se eliminó el amigo pero sigue aparecien- } \\
\text { do en mi lista de amigos, pero ya no puedo dar clic en el nombre." } \\
\text { Dos usuarios reportaron: "La aplicación FEIBook se detuvo". }\end{array}$ \\
\hline
\end{tabular}

Para corregir los defectos encontrados en la aplicación, se revisaron los resultados fallidos de los casos de prueba, así como la información de depuración generada por la plataforma Android en los dispositivos de los estudiantes, dando como resultado las siguientes actividades para realizar las correcciones:

- Para evitar los problemas de carga de la aplicación, se realizó la optimización de los recursos gráficos utilizados por la plataforma disminuyendo el peso de los mismos.

- La detención abrupta de la aplicación se solucionó disminuyendo la instanciación de objetos innecesarios.

- Para corregir el defecto de la lista de amigos, se implementó la notificación de cambios a la lista de amigos en la aplicación para refrescar la lista y no mostrar los amigos eliminados.

\section{Conclusiones}

En este trabajo se presentó el desarrollo de FEIBook versión móvil. FEIBook es una red social construida para llevar a cabo el seguimiento de egresados de los programas académicos de la Facultad de Estadística e Informática. Esta red social fue desarrollada empleando Elgg, un CMS que sigue el patrón MVC. Con el fin de aprovechar el acceso a dispositivos móviles que actualmente se tiene y facilitar así la actualización de los datos de los egresados en la red social, se propone realizar la versión móvil de FEIBook para el 
sistema operativo Android. Se busca entonces alguna manera de aprovechar la arquitectura tipo MVC de FEIBook, exponiendo los métodos a través de algún tipo de interfaz. Aunque Elgg cuenta con un mecanismo para exponer sus servicios, y existen comunidades con algunas soluciones, no existe alguna que sea compatible con FEIBook. Se construye entonces FEIBook_WebServices, un plugin (API REST) que se comunica directamente con el controlador de Elgg y permite hacer uso de toda a funcionalidad de la red social. Se desarrolla, además, la aplicación FEIBook móvil para Android, que usa los servicios expuestos por el plugin. Los resultados de las pruebas permiten asegurar que el plugin y la aplicación funcionan correctamente. La principal contribución de este trabajo es integrar las redes sociales y la tecnología móvil en una aplicación para el ámbito universitario. En [13] se presenta una revisión detallada de redes sociales en el ámbito universitario. Sin embargo, al momento de realizar este trabajo se desconoce la existencia de RSM en el ámbito universitario.

Los trabajos futuros propuestos a partir de este trabajo, son los siguientes:

En primer lugar mejorar la usabilidad de la interfaz gráfica de la aplicación. Aunque se diseñó y desarrolló una interfaz usable y accesible para los usuarios, la usabilidad no era un objetivo primario. Es así que, queda por realizar el diseño y desarrollo de una interfaz altamente usable para los usuarios de la red social.

Un segundo trabajo es que la aplicación móvil para Android permita subir y visualizar fotografías y videos así como interactuar en los grupos a los que se pertenece. Por último, queda pendiente realizar pruebas de seguridad, de desempeño y de resistencia que, por cuestiones de tiempo, no fue posible realizar en este trabajo.

\section{Referencias}

1. Barrón M.C.: Los estudios de seguimiento de egresados en el período 1992-2002, en Educación, trabajo, ciencia y tecnología, Colección la Investigación Educativa en México 1992-2002, libro 6. COMIE, México (2003)

2. Barrera Gallegos S.: Actualización de la Red Social de la Facultad de Estadística e Informática, Tesis de Licenciatura. Universidad Veracruzana, Xalapa, Ver. México (2013)

3. Buschmann, F., Rohnert, H., \& Stal, M: Pattern-oriented software architecture.Volume 1, a system of patterns, J. Wiley and Sons. New York (1996)

4. Elgg http://elgg.org

5. Engine Elgg Documentation http://docs.elgg.org/wiki/Engine/Views

6. All site plugins, themes, languages packs: The Elgg Communityt http://community.elgg.org/plugins

7. Forms+actions, Elgg Documentation http://learn.elgg.org/en/latest/guides/actions.html

8. González Jiménez B.: ReSoSE: Red Social para el Seguimiento de Egresados, Tesis de Licenciatura. Universidad Veracruzana, Xalapa, Ver. México (2012)

9. González-Jiménez B., Gontreras-Vega G., Cortés-Verdín M.:Redes Sociales para el Seguimiento de Egresados. En: Research in Computer Science, Special Issue: Advances in Intelligent 
Luis Abraham Zavaleta Ibarra, Gerardo Contreras Vega, Karen Cortés Verdín, et al.

and Information Technologies, vol. 60, pp.239-249, Centro de Investigación en Computación, Instituto Politécnico Nacional. México (2012)

10. InfoWorld http://www.infoworld.com/d/open-source/infoworld-announces-our-2008-bestopen-source-awards-065

11. Lane, N.: Mobile Social Networking, White Paper (2009). http://www.telecoms.com/files/2009/05/buongiorno_final-fmt_nl-3110-f.pdf

12. The Salzburg Academy on Media and Global Change. http://www.salzburgglobal.org/current/sa-b.cfm

13. Zavaleta Ibarra L. A.: Desarrollo de FEIBook versión móvil, Tesis de Licenciatura. Universidad Veracruzana, Xalapa, Ver. México (2014) 